\title{
SEMIAUTOMATIC ALGORITHM FOR THE INTERPRETATION OF THE BEDFORMS AND STATISTICAL ANALYSIS
}

\author{
LEONARDO SCHIPPA \& IRENE CAVALIERI \\ Department of Engineering, University of Ferrara, Italy
}

\begin{abstract}
This study presents a methodology to simplify the identification of the primary bedforms (mediumlarge dunes) present on a compound riverbed. The paper refers to a field study conducted on a straight free flow reach of an alluvial river (i.e. River Po in Italy) in January 2021. The surveys were carried out employing a multibeam echosounder which allows a large data collection, which was processed realizing river bed DTMs. The identification of the dunes along transects occurs through the implementation of a semiautomatic algorithm able to analyse the bed elevation profiles and to recognize the troughs and the crests of the primary dunes based on the threshold values of length obtained through to a spectral analysis. Once the dunes are recognized, characteristic parameters of the dunes (wavelength, wave height and steepness) are estimated, by which a statistical analysis is carried out to figure out the most frequently recurring dunes on the riverbed. The semiautomatic algorithm proves to be able to handle a large amount of data, providing a reliable interpretation of the riverbed and it is also consistent with results from previous studies. Starting from the present analysis, it is possible to develop the study of the bedforms kinematic that represents a key issue to quantify the volumes of solid bedload transport, in order to cross the typical limitations derived by the traditional bed load sampling methodologies.
\end{abstract}

Keywords: dunes bed, river bedform, river morphology, remote sensing riverbed.

\section{INTRODUCTION}

In recent decades, many Italian riverbeds have suffered significant morphologic changes. These changes, interpretable as a lowering of the riverbed quote, have drawn the attention both in the field of research and spatial planning. The need to apply a, monitoring, and programming activity to manage the evolution trends became evident, thus it is essential to support every planning activity with reliable forecast tools able to assess the evolution trends and river layout at medium to longer horizons, considering the changed hydrological situation due to climate change.

In alluvial river the bed forms often occur on the riverbed, they are complex and dynamic, and represent the most relevant feature governing flow resistance [1]. Complicating matters is the inherent variability of bedform size and shape in natural river systems. Sediment and water discharge conditions vary continuously in natural rivers causing bed morphology to be out of equilibrium with prevailing flow conditions [2]. In fact several field studies suggest that bedform disequilibrium is likely a standard phenomenon in natural rivers, and individual dunes in large sand rivers can have a wide range of geometry patterns corresponding to the same hydrometric condition [2], and it makes inconsistent most of the most accredited relationship among dune dimensions and hydraulic and sedimentary characteristics (e.g. [3], [4]). Therefore, even the estimation of flow resistance during engineering processes is not trivial, since it depends on the bed surface roughness and on the dimension of the bed forms, which in turn depend by flow parameters and sediment properties [5]. River bedforms can be distinguished in principle forms, the dunes, and secondary dunes, ripples and macroripples, which were found on the stoss slope and gentle lee slopes of large dunes [6]. These dunes usually increase in height and decrease in length towards the upper stoss of a large dune but 
may become less steep and more humpback in profile near the crest of the primary dune if the transition to upper-stage is approached locally in the crestal region crest [7].

The presence of the bed forms, their extension and geometry, influences the kinematic field, the flow resistance, the hydraulic efficiency of the section, and the stability of the fluvial infrastructures. The matter acquires particular importance when calculating the morphological changes they experience under changing flow conditions (e.g. steady and unsteady flow), or the downstream migration of dunes, which is of paramount importance to evaluate the bedload transport component. A popular approach involves calculating transport from the geometry and celerity of migrating bedforms, extracted from time series of bed elevation profiles [8]. It is well understandable that any of the former issue related to bed forms presence involve the knowledge of their geometry. To this purpose a random field approach to analyse spatial structure of bed undulation [9], or spectral analyses approach [10] may be applied.

A novel approach combining topographic, statistic and spectral analysis of large sand river bedforms is presented, and a semiautomatic algorithm aiming to identify bed form characteristic is implemented. To this purpose, a field campaign was launched in different hydrometric conditions along a reach of Po river (Italy) in June 2014, July 2014 and January 2021. The paper at hand particularly describes the last field surveying and shows preliminary results.

\section{STUDY AREA, METHODS AND SURVEYS}

\subsection{Study area}

The Po River, with a length of $652 \mathrm{~km}$ and a hydrographic basin of about 72,000 $\mathrm{km}^{2}$, is considered the most important Italian river and one of the principle European rivers. It is situated in norther Italy (Fig. 1(a)) and aided by the fact that its basin covers a quarter of the national territory, its waters represent a hydric resource for the irrigation and an important waterway.

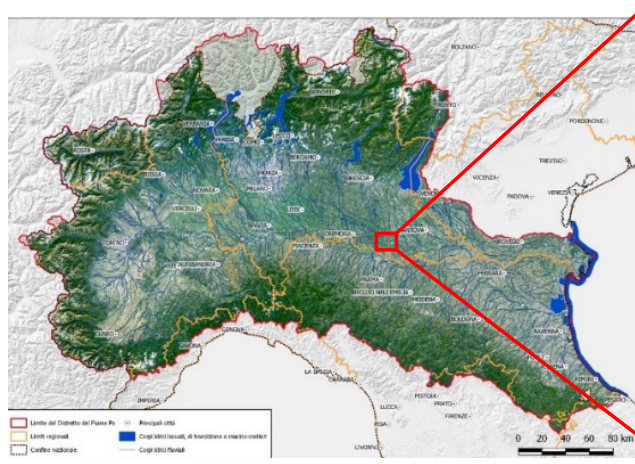

(a)

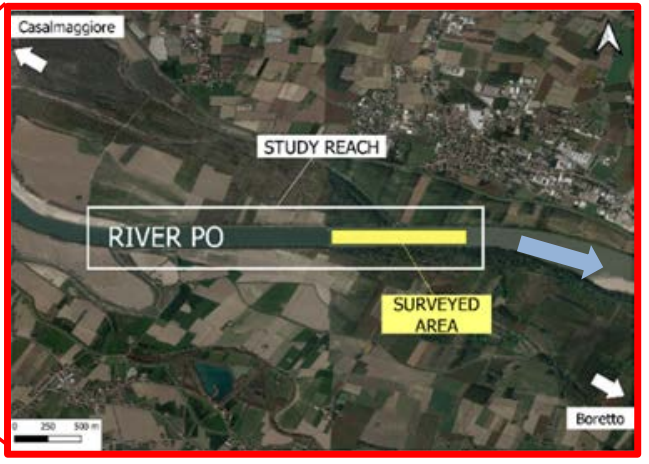

(b)

Figure 1: (a) River Po basin; and (b) Study reach and surveyed area.

The annual hydrological regime is characterized by two low water periods (winter and summer) and by two flood periods (late winter and spring) which are associated, respectively, to rainfall and to the snow melting processes from the mountain district of the basin. The 
minimum, medium and maximum annual discharges observed at the gauging station Pontelagoscuro are around $600,1,500$ and $5,000 \mathrm{~m}^{3} / \mathrm{s}$, respectively connected to flow peak of $10,000 \mathrm{~m}^{3} / \mathrm{s}$ and lower flow at $250 \mathrm{~m}^{3} / \mathrm{s}$.

The study reach (Fig.1(b)) is located $250 \mathrm{~km}$ upstream the delta and it is situated between two gauging stations, Casalmaggiore and Boretto. It is a single straight active channel reach having a length of $2.6 \mathrm{~km}$, a width of about $215 \mathrm{~m}$, and a mean bed slope of $0.15 \mathrm{~m} / \mathrm{km}$, lower than the ground slope (i.e. $0.26 \mathrm{~m} / \mathrm{km}$ ). The characteristic diameters of the bed sediment are $\mathrm{d}_{35}=0.35 \mathrm{~mm}, \mathrm{~d}_{50}=0.39 \mathrm{~mm}$, and $\mathrm{d}_{90}=0.76 \mathrm{~mm}$. In particular, the surveyed area (Fig. 1(b)) is $1 \mathrm{~km}$ long. Water level recording and stage-discharge relation are available for both gauging stations. The discharge duration (1943-2017) in the downstream station is $\mathrm{Q}_{10}=3,630 \mathrm{~m}^{3} / \mathrm{s}$, $\mathrm{Q}_{60}=1,810 \mathrm{~m}^{3} / \mathrm{s}, \mathrm{Q}_{91}=1,460 \mathrm{~m}^{3} / \mathrm{s}, \mathrm{Q}_{182}=933 \mathrm{~m}^{3} / \mathrm{s}, \mathrm{Q}_{355}=379 \mathrm{~m}^{3} / \mathrm{s}$.

\subsection{Methods and surveys}

The survey was carried out on 28th January 2021 thanks to a high-speed craft suitable for a high-speed hydrographic survey of the riverbed. It is equipped with a multibeam echosounds system MBES and a GPS system recording the actual position of each swat. According to the hydrometric level observed at the gauging station of Boretto and to the flow rate curve the mean discharge is about $\mathrm{Q}=1,327 \mathrm{~m}^{3} / \mathrm{s}$, furthermore the mean water depth on the surveyed area was $\mathrm{y}=5.90 \mathrm{~m}$ (Table 1$)$.

Table 1: Hydrometric conditions during the field campaign on the 28th January 2021, referring to starting and ending time of surveying.

\begin{tabular}{|l|c|c|c|c|}
\hline \multicolumn{4}{|c|}{ Hydrometric conditions $(\mathrm{y}=5.90 \mathrm{~m})$} \\
\hline & \multicolumn{2}{|c|}{ Starting time } & \multicolumn{2}{c|}{ Ending time } \\
\hline & $\begin{array}{c}\text { Water stage } \\
(\mathrm{m})\end{array}$ & $\begin{array}{c}\text { Discharge } \\
\left(\mathrm{m}^{3} / \mathrm{s}\right)\end{array}$ & $\begin{array}{c}\text { Water stage } \\
(\mathrm{m})\end{array}$ & $\begin{array}{c}\text { Discharge } \\
\left(\mathrm{m}^{3} / \mathrm{s}\right)\end{array}$ \\
\hline Boretto & $-0.23 \mathrm{~m}$ & $1,335 \mathrm{~m}^{3} / \mathrm{s}$ & $-0.27 \mathrm{~m}$ & $1,320 \mathrm{~m}^{3} / \mathrm{s}$ \\
\hline Casalmaggiore & $-1.54 \mathrm{~m}$ & & $-1.56 \mathrm{~m}$ & \\
\hline
\end{tabular}

The surveys started at fluvial harbour of Mezzani, sailing downstream in direction of Boretto to reach the surveying area. Two flanked swaths $50 \mathrm{~m}$ wide, and slightly overlapped over a streamwise distance of about $1,000 \mathrm{~m}$ were surveyed. The second survey of the same area was repeated in the same way after 40 minutes to gather the dune migration.

\section{BEDFORM IDENTIFICATION: DESCRIPTION OF THE ALGORITHM}

Once the surveys data were collected, the DTMs were processed. Fig. 2(a) represents the DTM obtained by a grid of $0.2 \mathrm{~m}$ surveyed points. Six longitudinal transects spaced few meters each other were defined and riverbed profiles were plotted, starting from the river bed bathymetry. A profile was collected for each line and for each time, so twelve profiles were analysed. The semiautomatic algorithm was applied to each profile and finally a unique sample was achieved. This sample allow the statistical analysis estimating the mean values, the modes, and the standard deviations.

Following the "discrete" approach, the river bottom is considered as a set of discrete twoor three-dimensional bedforms, which can be described by a set of parameters such as length, height, slope [11], thus every dune is theoretically simplified as a triangle identified by two troughs on the bottom and a crest on the top. 


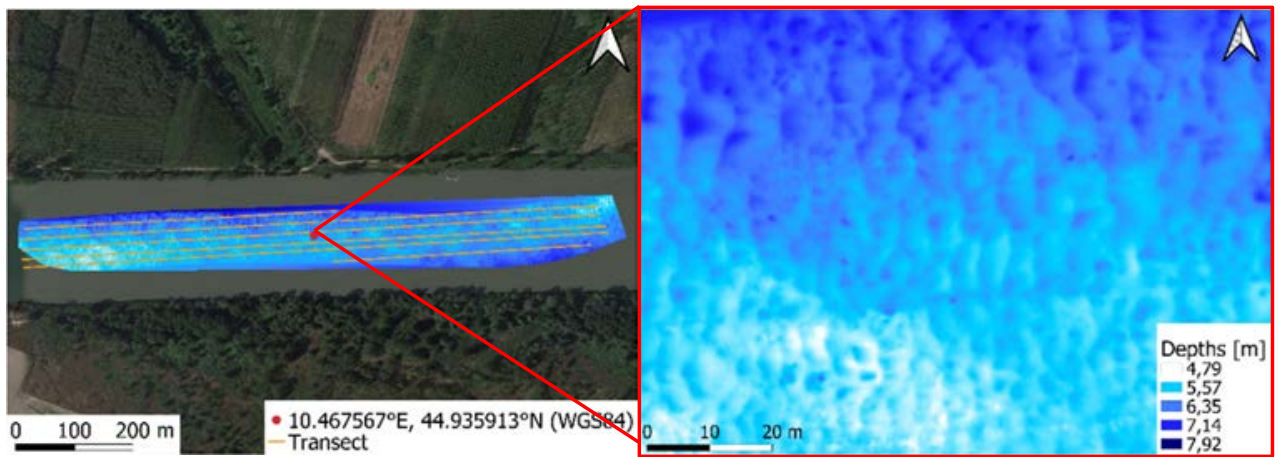

(a)

(b)

Figure 2: (a) DTM survey 28 January 2021; and (b) DTM detail.

A preliminary algorithm was implemented, which was able to identify all the local maxima and all the local minima along the profile producing a geometrical interpretation, that did not give an understandable representation of the bed undulation. In fact, secondary (smaller) forms superimposed to primary dunes are present, so losing the data connected to the existence of principal dunes. In fact, the result of the spectral analysis shows a signal characterized by a relevant spectral power around 4-6 m wavelength (Fig. 3).

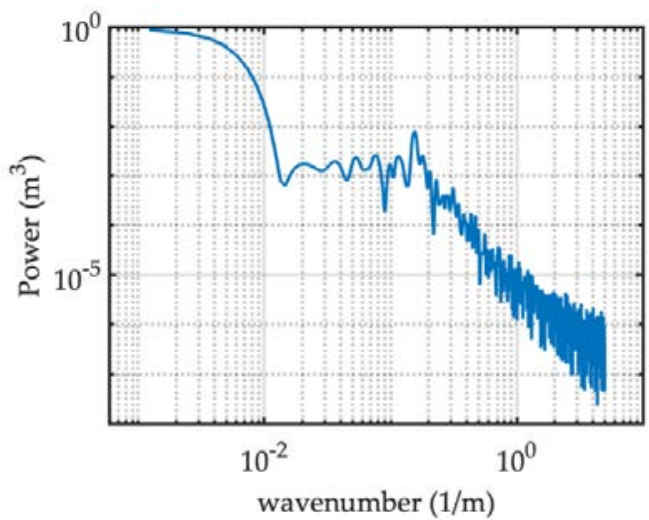

Figure 3: Single-sided spectrum for a representative longitudinal transect.

To overcome the problem, a novel semiautomatic algorithm to identify primary and secondary bed forms and giving a more truthful riverbed interpretation was implemented. Applying filtering parameters, it is possible to isolate those bedforms which are classified as principal forms. Filtering parameters are imposed according to the results of the spectral analysis, whose approach treats the riverbed as a continual moving wave field in which the forms may be described by wavenumber spectra. thus the algorithm was set using these last filtering values.

The semiautomatic algorithm allows to filter the bed elevation profiles which are complex, to recognize the principal bed forms. Based on the results of the spectral analysis, and 
according to [6], we may distinguish between ripples $(\lambda<0.6 \mathrm{~m})$ and small dunes $(0.6<\lambda<$ $5 \mathrm{~m})$ on one hand, and medium to large dunes $(5 \mathrm{~m}<\lambda)$ on the other hand. We consider the latter as the primary features of the river bed, and in our case they result $\lambda_{t}>4-6 \mathrm{~m}$, in relation to a wave amplitude $\Delta_{\mathrm{t}}>0.15 \mathrm{~m}$ as it results in the filtered bed profiles.

In the first place, the algorithm was applied to each transect along the swath. The proposed algorithm performs these next steps:

1. Bed elevation interpretation through the addition of the geometrical coordinates from the surveys and DTMs. The coordinates are imported to represents the bed elevation. Specifically, the transformation from height profile to bed relative elevation profile occurs.

2. Geometrical identification. Local maxima and local minima are located on the transect profile, thus every crest and every trough are identified (Fig. 4(a)).

3. Definition of the threshold values $\lambda_{t}$ and $\Delta_{t}$. According to the results of the spectral analysis, those values stand for the limit value which marks the clear separation between the existence of the secondary undulations $(\lambda<4-6 \mathrm{~m} ; \Delta<0.15 \mathrm{~m})$ or, on the contrary the presence of a dunes $(\lambda>4-6 \mathrm{~m} ; \Delta>0.15 \mathrm{~m})$. In this case the evaluations were carried out using $\lambda=4 \mathrm{~m}$.

4. Setting of a loop able to apply the above-mentioned filter according to threshold values. Scan of each bed form and recognizing only those forms corresponding to filter criterion. Finally, the identification of the filtered dunes through suitable crests and suitable troughs occurs (Fig. 4(b)).

5. In the end, dune (primary bed forms) geometric parameters are computed as following:

- Dune length. $\lambda$ : it represents the horizontal distance between two subsequent troughs along the longitudinal transect. Length $\lambda^{\prime}$ : it represents the horizontal distance between the crests two consecutive dunes. Length $\lambda_{\text {stoss }}$ : it represents the horizontal distance between the initial trough and the crest of a dune. Length $\lambda_{\text {lee }}$ it represents the horizontal distance between the crest of a dune and its final trough.

- Dune height. $\Delta_{\text {stoss: }}$ it represents the vertical distance between the upstream trough and the subsequent crest of a dune. $\Delta_{\text {lee }}$ : it represents the vertical distance between the crest of a dune and its downstream trough.

- Dune steepness. Stoss steepness is the ratio between $\Delta_{\text {stoss }}$ and $\lambda_{\text {stoss. }}$ Lee steepness it is the ratio between $\Delta_{\text {lee }}$ and $\lambda_{\text {lee. }}$. Representative steepness is the ratio between $\Delta_{\text {lee }}$ and $\lambda$.

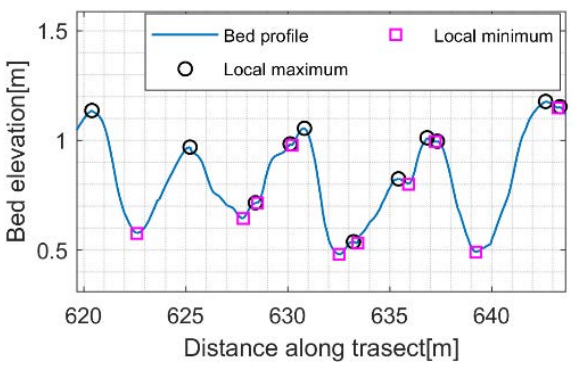

(a)

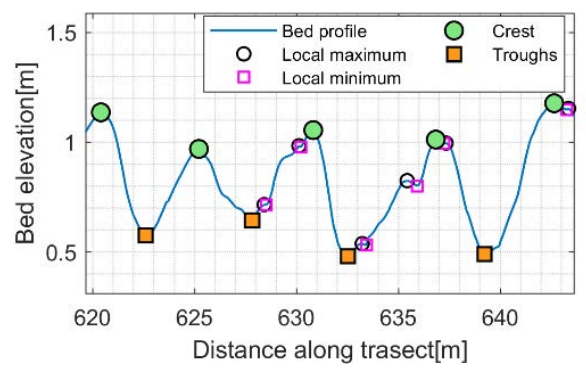

(b)

Figure 4: (a) Geometrical bed profile and local extreme location; (b) Filtered bed profile. Subsequent crest location $\bigcirc$, and through location $\square$, of the primary bed forms (i.e. dunes). 


\section{ALGORITHM'S APPLICATION, SENSITIVITY ANALYSIS AND DISCUSSION}

As a first step, a random subjective comparison between longitudinal transects extrapolated from the MBES and the filtered geometry has been carried out, confirming the consistency of the procedure. Then statistical analysis of the filtered geometry has been carried out assuming as a reference threshold value $\lambda_{t}=6 \mathrm{~m}$ as it derives from spectral analysis. Lognormal probability distribution function is depicted in Figs 5 and 6, whereas Fig. 7 plots the whole dataset coming from all the longitudinal transects.

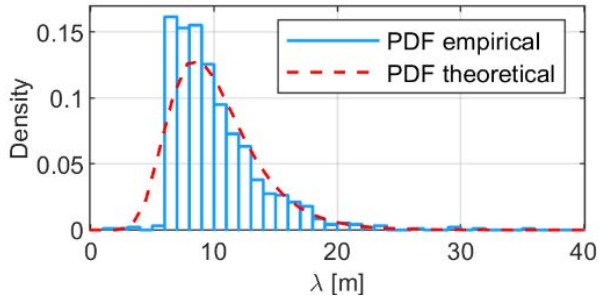

(a)

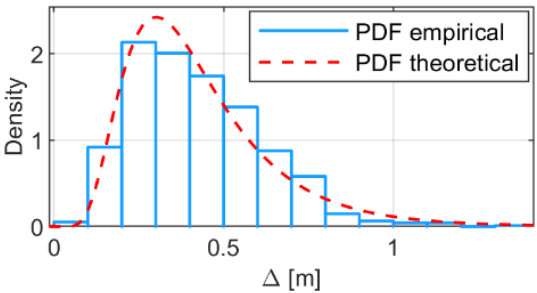

(b)

Figure 5: Empirical and theoretical PDF $\left(\lambda_{t}=6 \mathrm{~m}\right)$. (a) Dune length $\lambda$; and (b) Dune height $\Delta_{\text {lee. }}$

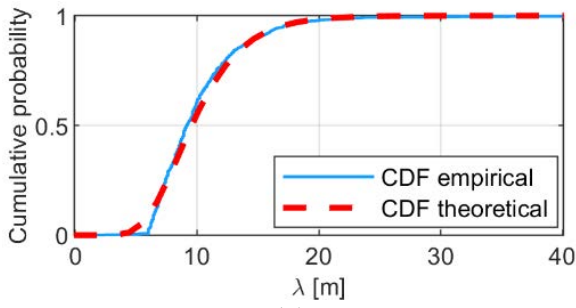

(a)

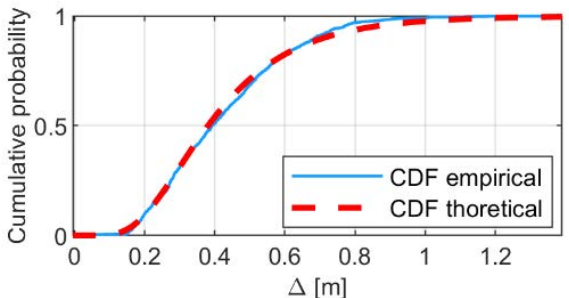

(b)

Figure 6: Empirical and theoretical cumulative distribution function CDF $\left(\lambda_{t}=6 \mathrm{~m}\right)$. (a) Dune length $\lambda$; and (b) Dune height $\Delta_{\text {lee. }}$.

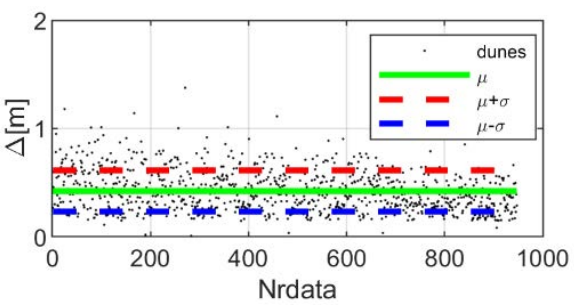

(a)

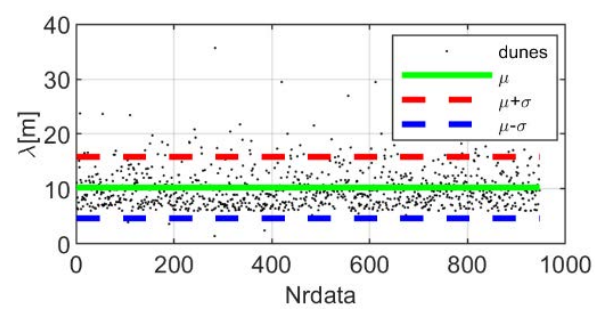

(b)

Figure 7: Statistical analysis $\left(\lambda_{\mathrm{t}}=6 \mathrm{~m}\right)$. (a) Dune length $\lambda$; and (b) Dune height $\Delta_{\text {lee. }}$.

Sensitivity analysis of the algorithm to the threshold values $\lambda_{t}$ and $\Delta_{t}$, has been carried out, taking advantage of statical analysis, and it is shown in Fig. 8 in terms of dune length $\lambda$ and 
dune height $\Delta_{\text {lee }}$. Table 2 reports the main statistical parameters of the characteristic dune dimensions based on a unique sample and calculated for the wave length threshold values $\lambda_{\mathrm{t}}=4 \mathrm{~m}, \lambda_{\mathrm{t}}=6 \mathrm{~m}$ and $\lambda_{\mathrm{t}}=8 \mathrm{~m}$. The frequency peak for $\lambda$ results equal to 5-6 m, 6-7 m and 9-10 $\mathrm{m}$ respectively. In the same way the peaks for $\lambda$ ' are equal to 5-6 $\mathrm{m}$ and 9-10 $\mathrm{m}$. The partial dune lengths $\lambda_{\text {stoss }}$ and $\lambda_{\text {lee }}$ result, respectively, equal to 2-4 m and 1-3 $\mathrm{m}\left(\lambda_{\mathrm{t}}=4 \mathrm{~m}\right)$, and equal to $2-5 \mathrm{~m}$ and $1-3 \mathrm{~m}\left(\lambda_{\mathrm{t}}=6 \mathrm{~m}\right.$ and $\left.\lambda_{\mathrm{t}}=8 \mathrm{~m}\right)$. Similarly, the process is repeated to estimate the wave height, the frequently occurring values can be observed between $0.2 \mathrm{~m}$ and $0.5 \mathrm{~m}$ in every case. Sensitivity analysis (Fig. 8) shows increasing lengths and heights values when threshold value increase, but the difference referred to their mean values are limited to $21 \%$ for lengths $\lambda$ and $4 \%$ for heights $\Delta_{\text {leee }}$.

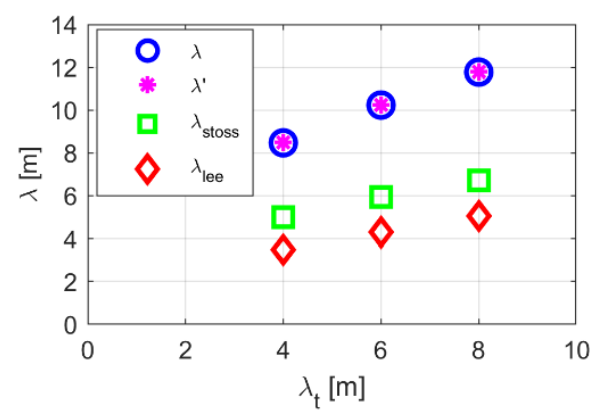

(a)

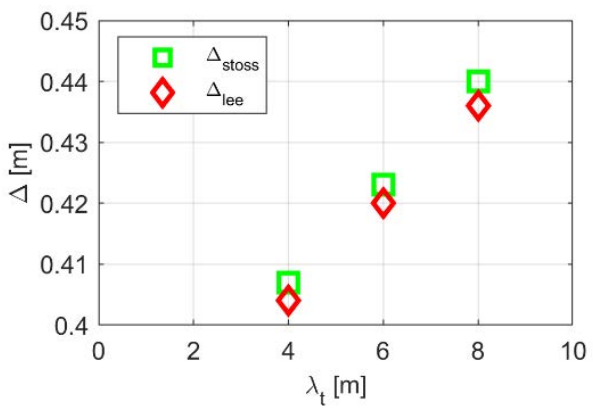

(b)

Figure 8: Sensitivity analysis. (a) Dune length; and (b) Dune height.

Table 2: Statistical parameters of the characteristic dune dimensions corresponding to different threshold value of $\lambda_{t}$. ( $\mu$ is mean value; $\sigma$ is standard deviation).

\begin{tabular}{|c|c|c|c|c|c|c|c|c|c|c|}
\hline & & $\lambda$ & $\Lambda^{\prime}$ & $\lambda_{\text {stoss }}$ & $\lambda_{\text {lee }}$ & $\Delta_{\text {stoss }}$ & $\Delta_{\text {lee }}$ & $\delta_{\text {stoss }}$ & $\delta_{\text {lee }}$ & $\Delta_{\text {lee }} / \lambda$ \\
\hline \multirow{2}{*}{$\lambda_{\mathrm{t}}=4 \mathrm{~m}$} & $\mu$ & 8.473 & 8.485 & 5.008 & 3.466 & 0.407 & 0.404 & 0.102 & 0.153 & 0.054 \\
\cline { 2 - 10 } & $\sigma$ & 5.357 & 5.795 & 5.014 & 2.535 & 0.181 & 0.186 & 0.057 & 0.086 & 0.026 \\
\hline \multirow{2}{*}{$\lambda_{\mathrm{t}}=6 \mathrm{~m}$} & $\mu$ & 10.236 & 10.247 & 5.935 & 4.301 & 0.423 & 0.420 & 0.091 & 0.136 & 0.045 \\
\cline { 2 - 10 } & $\sigma$ & 5.609 & 6.524 & 5.500 & 3.124 & 0.190 & 0.194 & 0.052 & 0.086 & 0.022 \\
\hline \multirow{2}{*}{$\lambda_{\mathrm{t}}=8 \mathrm{~m}$} & $\mu$ & 11.778 & 11.793 & 6.718 & 5.060 & 0.440 & 0.436 & 0.085 & 0.124 & 0.040 \\
\cline { 2 - 10 } & $\sigma$ & 5.750 & 6.981 & 5.866 & 3.492 & 0.194 & 0.199 & 0.051 & 0.084 & 0.018 \\
\hline
\end{tabular}

The steepness $\delta_{\text {stoss }}$ and $\delta_{\text {lee }}$ are focused, respectively, around $0.04-0.09$ and $0.07-0.10$ $\left(\lambda_{\mathrm{t}}=4 \mathrm{~m}\right), 0.05-0.08$ and $0.04-0.10\left(\lambda_{\mathrm{t}}=6 \mathrm{~m}\right)$, and $0.04-0.08$ and $0.03-0.09\left(\lambda_{\mathrm{t}}=8 \mathrm{~m}\right)$, total steepness $\delta$ result reasonably lower than the above-mentioned values and equal to $0.02-0.07$ $\left(\lambda_{\mathrm{t}}=4 \mathrm{~m}\right)$ and $0.02-0.05\left(\lambda_{\mathrm{t}}=6-8 \mathrm{~m}\right)$. Average dune steepness reduces increasing the threshold value $\lambda_{t}$, showing a moderate sensitivity to the choice of the threshold wavelength. On the opposite, wave amplitude (stoss or lee side calculated) is almost the same, and they are weakly sensitive to the choice of the threshold dune length value (Table 2). It is worth noting the influence of threshold value $\lambda_{t}$ on the dune length statistic. In fact, increasing $\lambda_{t}$ from $4 \mathrm{~m}$ to $8 \mathrm{~m}$, the average value of $\lambda$ (calculated trough-to-trough or crest-to-crest) increase by almost $40 \%$, and the standard deviation increase as well. The dune amplitude 
results almost the same, no matter of it is calculated stoss or lee side, and it moderately increases by $10 \%$ if we double $\lambda_{\mathrm{t}}$ from $4 \mathrm{~m}$ to $8 \mathrm{~m}$.

In particular, dune steepness $\delta_{\max }$ as a function of relative depth $Z=h / d_{s}$ is investigated (where $h$ is the local water depth, $d_{s}=0.430 \mathrm{~mm}$ is the mean sand diameter and $Z \in\left[1.16 \times 10^{4}\right.$ $\left.\left.-1.61 \times 10^{4}\right]\right)$. The variation of the dune steepness $\delta_{\max }$ related to flow intensity $\eta=\tau / \tau_{\mathrm{cr}}$ (where $\tau$ is the shear stress acting on the bed, and $\tau_{\mathrm{cr}}=15.0485 \mathrm{~N} / \mathrm{m}^{2}$ is its critical value calculated by Brownlie criterion [12]) is plotted in Fig. 9 along with data base of large sand rivers [13]. River Po data are consistent with large sand river database, statistical wise, and they confirm dune steepness are not easily featured by the approach originally proposed by Yalin and Karahan [14], mainly based on laboratory flume data. In other words what Yalin and Da Silva argued [13], about dune steepness in a real large sand river, seems to be confirmed by the field data herein presented. In fact, according to $Z>>1,000$ the maximum dune steepness values could be smaller than its theoretical value 0.06 , and the corresponding flow intensity may be larger than 16 .

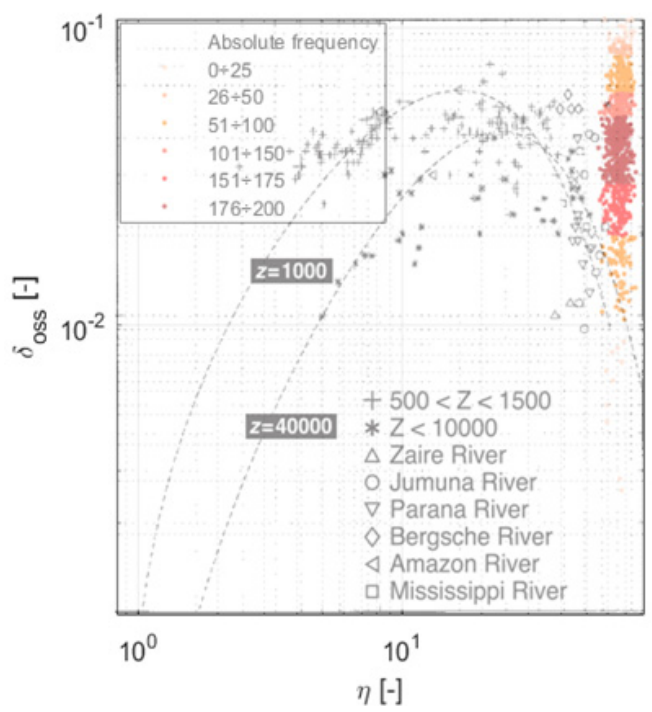

Figure 9: Dune steepness versus relative flow intensity. Po river data (coloured spots) are presented together with large sand river database (black spots) from [13].

Therefore, river Po data are plotted in Fig. 10 where the curve describes $\delta_{\max }$ derived by eqn (1) [13] and each dot represents a dune based on the calculated steepness $\delta_{\text {oss }}$ and its related $Z$.

$$
\delta_{\max }=0.00047 Z^{1.2} e^{-0.17 Z^{0.47}}+0.04\left(1-e^{-0.002 Z}\right) .
$$

Referring to the examined hydrodynamic conditions, and to the $Z$ range of the river Po field campaign, the maximum dune steepness calculated according to eqn (1) results $\delta_{\max }=0.04$. The average observed dune steepness results $\delta_{\text {oss }}=0.054 ; 0.054 ; 0.040$, depending on which $\lambda_{t}$ is considered (Table 2 ), and it is consistent with the theoretical value of $\delta_{\max }=0.04$. 


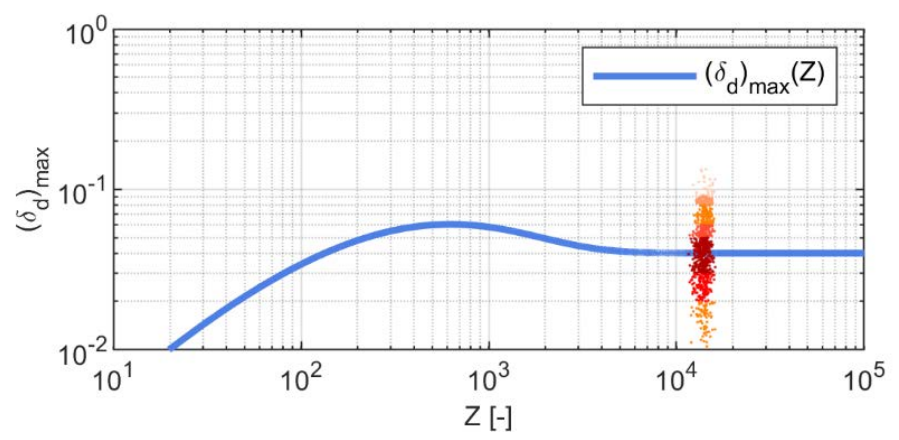

Figure 10: Theoretical maximum dune steepness (eqn (1)) and observed dune steepness.

To conclude this discussion session, it is worth to mention the potentiality of deriving dune kinematics by filtered dune geometry. Quantification of bedform migration by remote sensing has been studied for many years with limited success, both in river and shallow water sea environment, motivating some researchers to conclude that accurate observations require solid markers installed on the sea (river) bed. It introduces operational problems which can be hardly overcome in the natural environment. The main difficulty related to remote sensing seems to be collecting a sufficiently large number of small to medium dunes that will produce the necessary statistical parameters. Fig. 11 shows two MBES subsequent surveys repeated within an hour. The two bed elevation profiles have been overlapped in order to gather the dune migration and therefore being able to appreciate the dune kinematic, which is the standard point to modelling bedload transport, based on dune geometry. As it is evident from Fig. 11 crest and through migration is sharply defined, and the accuracy is favoured by the RTK-GPS surveying.

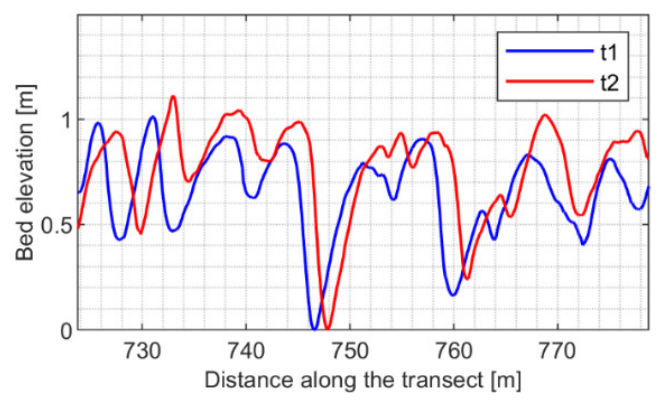

Figure 11: Overlapping of bed profiles obtained by MBES surveys carried out at $\mathrm{t} 1$ and $\mathrm{t} 2$.

\section{CONCLUSION}

The field campaign carried out on the middle course of the river Po and the application of the semiautomatic algorithm allowed to analyse the morphology of the riverbed. The algorithm has been tested on a large amount of data obtained with MBES in three survey campaigns, accounting for 39 longitudinal transects extrapolated from DTM covering about $10^{5} \mathrm{~m}^{2}$ of active riverbed. In the reported field campaign, dune lengths range of 4-9 $\mathrm{m}$, and dune heights consist of $0.2-0.5 \mathrm{~m}$ and the total steepness ranges between 0.03 and 0.06 for the investigated 
hydrometric conditions $\left(\mathrm{Q}=1,327 \mathrm{~m}^{3} / \mathrm{s}\right.$ and $\left.\mathrm{y}=5.90 \mathrm{~m}\right)$. Spectral analysis shows the prevalent wavenumber of the bed undulations, and it provides wavelength threshold value to be used for the filtered geometry. The algorithm developed plausible and truthful results in terms of filtered medium-large dune bed form, and it provides a correct interpretation of the riverbed, as it was confirmed by a random subjective comparison between filtered and original DTM river bed geometry along several random longitudinal transects. Sensitivity analysis demonstrates that dune steepness reduces increasing the threshold value $\lambda_{t}$, showing a moderate sensitivity to the choice of the threshold wavelength. On the opposite, wave amplitude (stoss or lee side calculated) is almost the same, and they are weakly sensitive to the choice of the threshold dune length value.

Further works are necessary to extend the field campaigns at higher flow conditions and during different rising period of selected flood to test any sensitivity to the hydrological condition.

\section{ACKNOWLEDGEMENTS}

The work has been realized thanks to the collaboration between Department of Engineering of University of Ferrara, AIPo and ARPAE. A grateful thanks to Alberto Agnetti, Luca Crose, and Paolo Piovani for the collaboration on field campaign organization, performing and data set analysing. Part of the work was developed during MSc thesis of Irene Cavalieri. The study was funded by AIPO contract 137/2019.

\section{REFERENCES}

[1] Schippa, L., Cilli, S., Ciavola, P. \& Billi, P., Dune contribution to flow resistance in alluvial rivers. Water, 11, 2094, 2019.

[2] Bradley, R.W. \& Venditti, J.G., Reevaluating dune scaling relations. Earth-Science Review Journal, 165, pp. 356-376, 2017.

[3] Karim, F., Bed configuration and hydraulic resistance in alluvial channel flows. Journal of Hydraulic Engineering. 121(1), pp. 15-25, 1995

[4] van Rijn, L.C., Sediment transport III: Bedforms and alluvial roughness. Journal of Hydraulic Engineering, 110, pp. 1733-1754, 1984.

[5] van Rijn, L., Equivalent roughness of alluvial bed. Journal of Hydraulic Engineering, pp. 1215-1218, 1982. https://doi.org/10.1061/(ASCE)0733-9429(1983)109:9(1250).

[6] Ashley, G.M., Classification of large-scale subaqueous bedforms: A new look at an old problem. J. Sedim. Petrol., 60, pp. 160-172, 1990.

[7] Carling, P.A., Golz, E., Orr, H.G. \& Radecki-Pawlikà, A., The morphodynamics of fluvial sand dunes in the River Rhine near Mainz, Germany. I. Sedimentology and morphology. Sedimentology, 47, pp. 227-252, 2000.

[8] Leary, K.C.P. \& Buscombe, D., Estimating sand bed load in rivers by tracking dunes: A comparison of methods based on bed elevation time series. Earth Surface Dynamics, 8, pp. 161-172, 2020.

[9] Aberle, J., Nikora, V., Henning, M., Ettmer, B. \& Hentschel, B., Statistical characterization of bed roughness due to bed forms: A field study in the Elbe River at Aken Germany. Water Resources Research, 46(3), 2010.

[10] Lefebvre, A., Ernstsen, V.\& Winter, C., Bedform characterization through 2D spectral analysis. Journal of Coastal Research, pp. 781-785, 2011.

[11] Nikora, V.I., Methods for quantitative description of channel bed-forms. Erosional and Channel Processes in Various Climatic Conditions, Moscow University, Moscow, pp. 327-328, 1987. (In Russian.)

[12] Brownlie, W.R., Compilation of alluvial channel data: Laboratory and field, 1981. 
[13] Yalin, M.S. \& Da Silva, A.M.F., Fluvial Processes, 2nd ed., Taylor \& Francis: London, pp. 51-57, 2017.

[14] Yalin, M.S. \& Karahan, E., Steepness of sedimentary dunes. Journal of the Hydraulics Division, ASCE, 105(4), pp. 381-392,1979. 